\title{
Improved Solution Enclosures for Over- and Underdetermined Interval Linear Systems ${ }^{\star}$
}

\author{
Evgenija D. Popova \\ Inst. of Mathematics \& Informatics, Bulgarian Academy of Sciences \\ Acad. G. Bonchev str., block 8, BG-1113 Sofia, Bulgaria \\ epopova@bio.bas.bg
}

\begin{abstract}
In this paper we discuss an inclusion method for solving rectangular (over- and under-determined) dense linear systems where the input data are uncertain and vary within given intervals. An improvement of the quality of the solution enclosures is described for both independent and parameter dependent input intervals. A fixed-point algorithm with result verification that exploits the structure of the problems to be solved is given. Mathematica functions for solving the discussed rectangular problems are developed and presented. Numerical examples illustrate the advantages of the proposed improved approach.
\end{abstract}

\section{Introduction}

Consider linear systems (1) where $A \in \mathbb{S}^{m \times n}, b \in \mathbb{S}^{m}$ and $\mathbb{S} \in\{\mathbb{R}, \mathbb{C}\}$,

$$
A \cdot x=b .
$$

For $m>n$, the linear system (1) is overdetermined and has no solution in general. In this case we are interested in a vector $\tilde{x} \in \mathbb{S}^{m}$ which minimizes the Euclidean norm $\|b-A \tilde{x}\|$ of the residual vector. If $m<n$ we have an underdetermined system. In general, there are infinitely many solutions and we look for a vector $\tilde{y} \in \mathbb{S}^{m}$ for which $A \tilde{y}=b$ and $\|\tilde{y}\|$ is minimal. If the rank of $A$ is maximal, the solution for both problems is uniquely determined. It is well known [9], that if

$$
\begin{aligned}
& m>n \text { and } \operatorname{rank}(A)=n \text { then } \tilde{x} \text { is the solution of } A^{H} A x=A^{H} b \\
& m<n \text { and } \operatorname{rank}(A)=m \text { then } \tilde{y}=A^{H} x, \text { where } A A^{H} x=b .
\end{aligned}
$$

However, $A^{H} A$ and $A A^{H}$ are in general ill-conditioned and furthermore not representable in the computer. In order to find guaranteed enclosures of the solutions to the above non-square problems, S. Rump [6] proposes to consider the following augmented square linear systems

$$
\begin{aligned}
&\left(\begin{array}{cc}
A & -I \\
0 & A^{H}
\end{array}\right) \cdot\left(\begin{array}{l}
x \\
y
\end{array}\right)=\left(\begin{array}{l}
b \\
0
\end{array}\right) \quad \text { for } m>n, \\
&\left(\begin{array}{cc}
A^{H} & -I \\
0 & A
\end{array}\right) \cdot\left(\begin{array}{l}
x \\
y
\end{array}\right)=\left(\begin{array}{l}
0 \\
b
\end{array}\right) \quad \text { for } m<n,
\end{aligned}
$$

\footnotetext{
* This work was supported by the Bulgarian National Science Fund under grant No. MM-1301/03.

I. Lirkov, S. Margenov, J. Waśniewski (Eds.): LSSC 2005, LNCS 3743, pp.305-312, 2006. (C) Springer-Verlag Berlin Heidelberg 2006
} 
instead of solving (2), (3). It is shown in [6] as a direct consequence that Rump's fixed-point inclusion method for square systems can be used to verify solvability of the over-/underdetermined problem, the maximum $\operatorname{rank}=\min (m, n)$ of the matrix $A$, and to produce guaranteed bounds for the uniquely determined solution. The method is naturally extendable to interval and complex interval matrices $[A] \in \mathbb{I S}^{m \times n}$ and vectors $[b] \in \mathbb{I S}^{m}$.

Following this approach, over-/underdetermined linear system solvers with result verification were implemented in the environments of PASCAL-XSC [2] and C-XSC [1]. For problems with interval (real or complex) input data these implementations are based on solving square interval linear systems (4), (5) assuming that all the elements vary independently in their intervals. A close look at the structure of the matrices in the systems (4), (5) shows that each element of the matrix $A$ appears twice in the augmented square matrix which means that this matrix involves dependencies. As is well-known "the dependency problem" in interval analysis causes severe overestimation of the corresponding solution set and may lead to an interval iteration matrix involving singular ones.

In this paper we derive an algorithm for sharp enclosure of the solution set to the square linear system used as a basis for the solution of an over/underdetermined interval (real or complex) linear problem (2), (3). The proposed algorithm improves the approach used in $[1,2]$ by taking into account the dependencies in the augmented matrix in order to improve the sharpness of the solution enclosure, and by using block matrix computations, related to the special structure of the matrix, to reduce the number of floating-point operations.

The following notations are used. $\mathbb{I R}, \mathbb{I} \mathbb{C}$ are the sets of real, resp. complex intervals. We assume that the reader is familiar with basic results of interval analysis (cf. [3]). For an interval $[a]:=\left[a^{-}, a^{+}\right] \in \mathbb{R}, \operatorname{mid}([a]):=\left(a^{-}+a^{+}\right) / 2$ is the mid point of $[a]$. By $\mathbb{I S}^{n}, \mathbb{I S}^{m \times n}$ denote the sets of interval (real or complex) $n$-vectors, resp. $m \times n$ matrices. For $\Sigma \subseteq \mathbb{R}^{n}$ the interval hull $\diamond(\Sigma)$ is defined by $\diamond(\Sigma):=\bigcap\left\{[w] \in \mathbb{R}^{n} \mid \Sigma \in[w]\right\} . A^{H}$ denotes the Hermitian matrix of a matrix $A$ (i.e. the transposed matrix in the real, resp. real interval case). $I$ is the identity matrix. An interval matrix $[A]$ has rank $n$ if every $A \in[A]$ has rank $n$.

\section{Theory and Algorithm}

Let $[A] \in \mathbb{S}^{m \times n},[b] \in \mathbb{I} \mathbb{S}^{m}$, where $\mathbb{S} \in\{\mathbb{R}, \mathbb{C}\}$, and $m \neq n$.

If $m>n$ and $\operatorname{rank}([A])=n$, define the set

$$
\begin{aligned}
\Sigma_{m>n} & :=\left\{\tilde{x} \in \mathbb{S}^{n} \mid \exists A \in[A], \exists b \in[b], \tilde{x}=\operatorname{argmin}_{x \in \mathbb{S}^{n}}\|b-A x\|\right\} \\
& =\left\{x \in \mathbb{S}^{n} \mid \exists A \in[A], \exists b \in[b], A^{H} A x=A^{H} b\right\} .
\end{aligned}
$$

If $m<n$ and $\operatorname{rank}([A])=m$, define the set

$$
\begin{aligned}
\Sigma_{m<n} & :=\left\{\tilde{y} \in \mathbb{S}^{n} \mid(\exists A \in[A])(\exists b \in[b])\left(\tilde{y}=\operatorname{argmin}_{y \in \mathbb{S}^{n}}\|y\|, A y=b\right)\right\} \\
& =\left\{y \in \mathbb{S}^{n} \mid(\exists A \in[A])(\exists b \in[b])\left(y=A^{H} x, A A^{H} x=b\right)\right\} .
\end{aligned}
$$


We are interested in finding verified enclosures of the solution sets $(6),(7)$ whenever they are bounded, that is interval vectors $[x],[y] \in \mathbb{I S}^{n}$ such that $[x] \supseteq \diamond\left(\Sigma_{m>n}\right)$ and $[y] \supseteq \diamond\left(\Sigma_{m<n}\right)$. The following theorems permit to calculate very sharp bounds to the solution sets (6), (7) and to estimate the sharpness of the calculated bounds. Define

$$
\begin{array}{lll}
\text { (o) } \quad[B]:=\left(\begin{array}{cc}
{[A]} & -I \\
0 & {[A]^{H}}
\end{array}\right), & {[v]:=([b], 0)^{\top} ;} \\
\text { (u) } \quad[B]:=\left(\begin{array}{cc}
{[A]^{H}-I} \\
0 & {[A]}
\end{array}\right), & {[v]:=(0,[b])^{\top} .}
\end{array}
$$

Theorem 1. Let $[A] \in \mathbb{I}^{m \times n},[b] \in \mathbb{I}^{m}, m>n$. Define $[B] \in \mathbb{I S}^{(m+n) \times(m+n)}$, $[v] \in \mathbb{I}^{m+n}$ to be the square matrix, resp. the vector in (8) and let $R \in$ $\mathbb{S}^{(m+n) \times(m+n)}, \tilde{u} \in \mathbb{S}^{m+n}$.

1) Let $[z] \in \mathbb{I S}^{m+n}$ be defined by

$$
\begin{array}{r}
{\left.\left[z_{i}\right]\right|_{i=1} ^{m+n}:=\sum_{\mu=1}^{m} r_{i \mu}\left(\left[b_{\mu}\right]+\tilde{u}_{n+\mu}\right)-\sum_{\mu=1}^{m} \sum_{\nu=1}^{n}\left(r_{i \mu} \tilde{u}_{\nu}+r_{i, m+\nu} \tilde{u}_{n+\mu}\right) \operatorname{Re}\left(\left[a_{\mu \nu}\right]\right)} \\
-\sum_{\mu=1}^{m} \sum_{\nu=1}^{n}\left(r_{i \mu} \tilde{u}_{\nu}-r_{i, m+\nu} \tilde{u}_{n+\mu}\right) \operatorname{Im}\left(\left[a_{\mu \nu}\right]\right)
\end{array}
$$

then $[z]=\diamond(\{R \cdot(v-B \tilde{u}) \mid B \in[B], v \in[v]\})$.

2) Let $[C] \in \mathbb{I} \mathbb{S}^{(m+n) \times(m+n)}$ is defined by $[C]:=I-R \cdot[B]$ and let $[u] \in \mathbb{I S}^{m+n}$. Define $[w] \in \mathbb{I S}^{m+n}$ by

$\left.\left[w_{i}\right]\right|_{i=1} ^{m+n}:=\{[z]+[C] \cdot[u u]\}_{i}$, where $[u u]:=\left(\left[w_{1}\right], \ldots,\left[w_{i-1}\right],\left[u_{i}\right], \ldots,\left[u_{m+n}\right]\right)^{\top}$

If $[w] \varsubsetneqq[u]$, then every matrix $A \in[A]$ has a full rank $n$, and for every $A \in[A]$, $b \in[b]$ with $\hat{x}$ minimizing $\|b-A x\|$, the unique solution $\hat{x}$ satisfies $\hat{x} \in \tilde{x}+[x]$, where $\tilde{x}$ and $[x]$ are the first $n$ components of $\tilde{u}$, resp. $[w]$.

3) With $[\Delta]:=[C] \cdot[w] \in \mathbb{I S}^{m+n}$ and $[x x] \in \mathbb{I S}^{n}$ defined by

$$
\left[x x_{i}\right]:=\left[\inf \left(\left[z_{i}\right]\right)+\sup \left(\left[\Delta_{i}\right]\right), \sup \left(\left[z_{i}\right]\right)+\inf \left(\left[\Delta_{i}\right]\right)\right], \quad 1 \leqq i \leqq n,
$$

the following inner and outer estimations hold true

$$
\tilde{x}+[x x] \subseteq \diamond\left(\Sigma_{m>n}\right) \subseteq \tilde{x}+[x] .
$$

Proof. 1) Let us represent $R$ by a block structure $R=\left(R^{1}, R^{2}\right)$, where

$$
\begin{aligned}
R^{1}=\left(r_{i \mu}^{1}\right):=\left(r_{i \mu}\right), \quad R^{2}=\left(r_{i \nu}^{2}\right):=\left(r_{i, m+\nu}\right), & 1 \leq \mu \leq m, 1 \leq \nu \leq n, \\
& 1 \leq i \leq m+n .
\end{aligned}
$$

Since for arbitrary $A \in[A], b \in[b]$ we have

$$
\begin{aligned}
z & :=R(v-B \cdot \tilde{u})=R^{1} \cdot b-\left(R^{1}, R^{2}\right) \cdot\left(\begin{array}{c}
A \tilde{u}^{1}-\tilde{u}^{2} \\
A^{H} \tilde{u}^{2}
\end{array}\right) \\
& =R^{1} \cdot b-\left(R^{1}, R^{2}\right) \cdot\left(\begin{array}{l}
A \tilde{u}^{1}-\tilde{u}^{2} \\
\left(\tilde{u}^{2 \top} \bar{A}\right)^{\top}
\end{array}\right)
\end{aligned}
$$




$$
\begin{aligned}
& R^{1} \cdot b-R^{1} \cdot\left(\begin{array}{c}
\sum_{\nu=1}^{n} a_{\mu \nu} \tilde{u}_{\nu}^{1}-\tilde{u}_{\mu}^{2} \\
\vdots \\
\mu=1, \ldots, m
\end{array}\right)-R^{2} \cdot\left(\begin{array}{c}
\sum_{\mu=1}^{m} \bar{a}_{\mu \nu} \tilde{u}_{\mu}^{2} \\
\vdots \\
\nu=1, \ldots, n
\end{array}\right) \\
&\left.z_{i}\right|_{i=1} ^{m+n}= \sum_{\mu=1}^{m} r_{i \mu} b_{\mu}-\sum_{\mu=1}^{m} r_{i \mu}^{1} \sum_{\nu=1}^{n} a_{\mu \nu} \tilde{u}_{\nu}^{1}+\sum_{\mu=1}^{m} r_{i \mu}^{1} \tilde{u}_{\mu}^{2}-\sum_{\nu=1}^{n} r_{i \nu}^{2} \sum_{\mu=1}^{m} \tilde{u}_{\mu}^{2} \bar{a}_{\mu \nu} \\
&= \sum_{\mu=1}^{m} r_{i \mu}\left(b_{\mu}+\tilde{u}_{n+\mu}\right)-\sum_{\mu=1}^{m} \sum_{\nu=1}^{n}\left(r_{i \mu} \tilde{u}_{\nu}+r_{i, m+\nu} \tilde{u}_{n+\mu}\right) \operatorname{Re}\left(a_{\mu \nu}\right) \\
&-\sum_{\mu=1}^{m} \sum_{\nu=1}^{n}\left(r_{i \mu} \tilde{u}_{\nu}-r_{i, m+\nu} \tilde{u}_{n+\mu}\right) \operatorname{Im}\left(a_{\mu \nu}\right)
\end{aligned}
$$

then 1) follows by a Theorem by Moore [3] because in (10) each interval variable occurs only once and to the first power. 2) follows from the fixed-point verification theory for non-square systems [6] and for parametric systems [8]. 3) is a consequence of the theory for estimating the quality of an outer enclosure [7].

Define another block structure for $R=\left(R^{3}, R^{4}\right)$, where $1 \leq i \leq m+n$ and

$$
R^{3}=\left(r_{i \nu}^{3}\right):=\left(r_{i \nu}\right), \quad R^{4}=\left(r_{i \mu}^{4}\right):=\left(r_{i, n+\mu}\right), \quad 1 \leq \nu \leq n, 1 \leq \mu \leq m
$$

Deriving analogously the expression which gives the exact range for the residuum

$$
\begin{aligned}
z:= & R(v-B \cdot \tilde{u})=R^{4} \cdot b-\left(R^{3}, R^{4}\right) \cdot\left(\begin{array}{c}
A^{H} \tilde{u}^{1}-\tilde{u}^{2} \\
A \tilde{u}^{2}
\end{array}\right) \\
= & R^{4} \cdot b-R^{3} \cdot\left(\begin{array}{c}
\sum_{\mu=1}^{m} \bar{a}_{\mu \nu} \tilde{u}_{\mu}^{1}-\tilde{u}_{\nu}^{2} \\
\vdots \\
\nu=1, \ldots, n
\end{array}\right)-R^{4} \cdot\left(\begin{array}{c}
\sum_{\nu=1}^{n} \bar{a}_{\mu \nu} \tilde{u}_{\nu}^{2} \\
\vdots \\
\mu=1, \ldots, m
\end{array}\right), \\
\left.z_{i}\right|_{i=1} ^{m+n}= & \sum_{\mu=1}^{m} r_{i \mu}^{4} b_{\mu}-\sum_{\nu=1}^{n} r_{i \nu}^{3} \sum_{\mu=1}^{m} \bar{a}_{\mu \nu} \tilde{u}_{\mu}^{1}+\sum_{\nu=1}^{n} r_{i \nu}^{3} \tilde{u}_{\nu}^{2}-\sum_{\mu=1}^{m} r_{i \mu}^{4} \sum_{\nu=1}^{n} a_{\mu \nu} \tilde{u}_{\nu}^{2} \\
= & \sum_{\mu=1}^{m} r_{i, n+\mu} b_{\mu}-\sum_{\mu=1}^{m} \sum_{\nu=1}^{n}\left(r_{i \nu} \tilde{u}_{\mu}+r_{i, n+\mu} \tilde{u}_{m+\nu}\right) \operatorname{Re}\left(a_{\mu \nu}\right) \\
& +\sum_{\nu=1}^{n} r_{i \nu} \tilde{u}_{m+\nu}+\sum_{\mu=1}^{m} \sum_{\nu=1}^{n}\left(r_{i \nu} \tilde{u}_{\mu}-r_{i, n+\mu} \tilde{u}_{m+\nu}\right) \operatorname{Im}\left(a_{\mu \nu}\right),
\end{aligned}
$$

we proof the following theorem for the underdetermined case.

Theorem 2. Let $[A] \in \mathbb{I} \mathbb{S}^{m \times n},[b] \in \mathbb{I} \mathbb{S}^{m}, m<n$ and define $[B] \in \mathbb{I} \mathbb{S}^{(m+n) \times(m+n)}$, $[v] \in \mathbb{I S}^{m+n}$ to be the square matrix, resp. the vector in (9) and let $R \in$ $\mathbb{S}^{(m+n) \times(m+n)}, \tilde{u} \in \mathbb{S}^{m+n}$.

1) Let $[z] \in \mathbb{I S}^{m+n}$ be defined by 


$$
\begin{array}{r}
{\left.\left[z_{i}\right]\right|_{i=1} ^{m+n}:=\sum_{\mu=1}^{m} r_{i, n+\mu} b_{\mu}+\sum_{\nu=1}^{n} r_{i \nu} \tilde{u}_{m+\nu}-\sum_{\mu=1}^{m} \sum_{\nu=1}^{n}\left(r_{i \nu} \tilde{u}_{\mu}+r_{i, n+\mu} \tilde{u}_{m+\nu}\right) \operatorname{Re}\left(a_{\mu \nu}\right)} \\
+\sum_{\mu=1}^{m} \sum_{\nu=1}^{n}\left(r_{i \nu} \tilde{u}_{\mu}-r_{i, n+\mu} \tilde{u}_{m+\nu}\right) \operatorname{Im}\left(a_{\mu \nu}\right), \quad \text { (13) }
\end{array}
$$

then $[z]=\diamond(\{R \cdot(v-B \tilde{u}) \mid B \in[B], v \in[v]\})$.

2) Let $[C] \in \mathbb{I} \mathbb{S}^{(m+n) \times(m+n)}$ is defined by $[C]:=I-R \cdot[B]$ and let $[u] \in \mathbb{I S}^{m+n}$. Define $[w] \in \mathbb{I S}^{m+n}$ by

$\left.\left[w_{i}\right]\right|_{i=1} ^{m+n}:=\{[z]+[C] \cdot[u u]\}_{i}$, where $[u u]:=\left(\left[w_{1}\right], \ldots,\left[w_{i-1}\right],\left[u_{i}\right], \ldots,\left[u_{m+n}\right]\right)^{\top}$

If $[w] \varsubsetneqq[u]$, then every matrix $A \in[A]$ has a full rank $m$, and for every $A \in[A]$, $b \in[b]$ with $A \hat{y}=b$ and $\hat{y}=\min \|y\|$, the unique solution $\hat{y}$ satisfies $\hat{y} \in \tilde{y}+[y]$, where $\tilde{y}$ and $[y]$ are the last $n$ components of $\tilde{u}$, resp. $[w]$.

3) With $[\Delta]:=[C] \cdot[w] \in \mathbb{I S}^{m+n}$ and $[y y] \in \mathbb{I} \mathbb{S}^{n}$ defined by

$$
\left[y y_{i}\right]:=\left[\inf \left(\left[z_{i}\right]\right)+\sup \left(\left[\Delta_{i}\right]\right), \sup \left(\left[z_{i}\right]\right)+\inf \left(\left[\Delta_{i}\right]\right)\right], \quad m+1 \leqq i \leqq m+n,
$$

the following inner and outer estimations hold true

$$
\tilde{y}+[y y] \subseteq \diamond\left(\Sigma_{m<n}\right) \subseteq \tilde{y}+[y] .
$$

Now, the following algorithm computes guaranteed enclosures of the solution sets (6), (7), for $[A] \in \mathbb{R}^{m \times n},[b] \in \mathbb{R}^{m}, m \neq n$.

1. Initialization. $\check{b}:=\operatorname{mid}([b]) ; \check{A}:=\operatorname{mid}([A])$;

Compose $\check{B}$ corresponding to (4), (5); Compute $R \approx \check{B}^{-1} \in \mathbb{R}^{(m+n) \times(m+n)}$.

Decompose $R$ into blocks defined by (11), (12).

2. Compute the approximate mid-point solution $\tilde{u}=R \check{v}$, where

$$
\text { (o) } \quad \tilde{u}=R^{1} \check{b}, \quad(u) \quad \tilde{u}=R^{4} \check{b} .
$$

3. Enclosure for the residuum, where for $1 \leq i \leq m+n$
(o) $\left[z_{i}\right]=\sum_{\mu=1}^{m} r_{i \mu}\left(\left[b_{\mu}\right]+\tilde{u}_{n+\mu}\right)-\sum_{\mu=1}^{m} \sum_{\nu=1}^{n}\left(r_{i \mu} \tilde{u}_{\nu}+r_{i, m+\nu} \tilde{u}_{n+\mu}\right)\left[a_{\mu \nu}\right]$;
(u) $\left[z_{i}\right]=\sum_{\mu=1}^{m} r_{i, n+\mu}\left[b_{\mu}\right]+\sum_{\nu=1}^{n} r_{i \nu} \tilde{u}_{m+\nu}-\sum_{\mu=1}^{m} \sum_{\nu=1}^{n}\left(r_{i \nu} \tilde{u}_{\mu}+r_{i, n+\mu} \tilde{u}_{m+\nu}\right)\left[a_{\mu \nu}\right]$.

4. Enclosure for the iteration matrix:
(o) $[C]=I-\left(R^{1}[A], R^{2}[A]^{\top}-R^{1}\right)$,
(u) $[C]=I-\left(R^{3}[A]^{\top}, R^{4}[A]-R^{3}\right)$.

5. Verification

$[u u]:=[z]$;

repeat

$$
\left[u_{\text {new }}\right]:=[u u]:=\operatorname{blow}([u u], \varepsilon)
$$

for $i=1$ to $m+n$ do $\left[u u_{i}\right]:=\left[z_{i}\right]+\left[C_{i}\right] \cdot[u u]$

until $[u u] \varsubsetneqq\left[u_{\text {new }}\right]$ or max iteration exceeded 


\section{If $[u u] \varsubsetneqq\left[u_{\text {new }}\right]$ then}

a unique solution $u$ exists and $u \in \tilde{u}+[u u]$, hence

$\diamond\left(\Sigma_{m>n}\right) \subseteq \tilde{x}+[x]$, where $\tilde{x}$ and $[x]$ are the first $n$ components of $\tilde{u}$, resp. [uu]; $\diamond\left(\Sigma_{m<n}\right) \subseteq \tilde{y}+[y]$, where $\tilde{y}$ and $[y]$ are the last $n$ components of $\tilde{u}$, resp. [uu].

else algorithm fails, matrix $[B]$ is ill conditioned or singular.

The above algorithm can be rigorously implemented on a computer. Function blow $([u u], \varepsilon)$ (for the definition see $[1,2,4]$ ) does the so-called $\varepsilon$-inflation which blows up the intervals somewhat, in order to catch the nearby fixed point. Some modifications of the above algorithm can be done in order to make it more robust in the case of almost singular matrices [2]. A cheap method for computing an estimation of the sharpness of the calculated outer bounds is presented in [4].

\section{Parameter Dependence}

Let us suppose now that the elements of the matrix $A$ and the vector $b$ depend affine-linearly on a parameter vector $p=\left(p_{1}, \ldots, p_{k}\right)^{\top}, p \in[p] \in \mathbb{R}^{k}$. In this case $A(p)$ and $b(p)$ have the following factored representation

$$
\begin{aligned}
A(p) & =A^{(0)}+A^{(1)} p_{1}+\cdots+A^{(k)} p_{k}, & & A^{(j)} \in \mathbb{R}^{m \times n}, \\
b(p) & =b^{(0)}+b^{(1)} p_{1}+\cdots+b^{(k)} p_{k}, & & b^{(j)} \in \mathbb{R}^{m}, j=0,1, \ldots, k .
\end{aligned}
$$

For $1 \leq i \leq m+n$ we have the following exact ranges for the residuum

$$
\begin{array}{r}
{\left[z_{i}\right]=\sum_{\mu=1}^{m}\left(r_{i \mu} \tilde{u}_{n+\mu}+r_{i \mu} b_{\mu}^{(0)}-\sum_{\nu=1}^{n}\left(r_{i \mu} \tilde{u}_{\nu}+r_{i, m+\nu} \tilde{u}_{n+\mu}\right) a_{\mu \nu}^{(0)}\right)} \\
+\sum_{j=1}^{k}\left[p_{j}\right]\left(\sum_{\mu=1}^{m} r_{i \mu} b_{\mu}^{(j)}-\sum_{\mu=1}^{m} \sum_{\nu=1}^{n}\left(r_{i \mu} \tilde{u}_{\nu}+r_{i, m+\nu} \tilde{u}_{n+\mu}\right) a_{\mu \nu}^{(j)}\right), \\
{\left[z_{i}\right]=\sum_{\mu=1}^{m} r_{i, n+\mu} b_{\mu}^{(0)}+\sum_{\nu=1}^{n} r_{i \nu} \tilde{u}_{m+\nu}-\sum_{\mu=1}^{m} \sum_{\nu=1}^{n}\left(r_{i \nu} \tilde{u}_{\mu}+r_{i, n+\mu} \tilde{u}_{m+\nu}\right) a_{\mu \nu}^{(0)}} \\
+\sum_{j=1}^{k}\left(\sum_{\mu=1}^{m} r_{i, n+\mu} b_{\mu}^{(j)}-\sum_{\mu=1}^{m} \sum_{\nu=1}^{n}\left(r_{i \nu} \tilde{u}_{\mu}+r_{i, n+\mu} \tilde{u}_{m+\nu}\right) a_{\mu \nu}^{(j)}\right)\left[p_{j}\right] .
\end{array}
$$

The improved enclosure of the iteration matrix $C$, for $m>n$ e.g., is

$[C]=I-\left(R^{1} A^{(0)}+\sum_{j=1}^{k}\left[p_{j}\right]\left(R^{1} A^{(j)}\right), R^{2} A^{(0) \top}-R^{1}+\sum_{j=1}^{k}\left[p_{j}\right]\left(R^{2} A^{(j) \top}\right)\right)$.

An implementation of the methods/algorithm, presented in Section 2, which exploits the above given expressions for the exact ranges of the residual and the iteration matrix will produce very sharp bounds for the solution sets to over- and underdetermined linear systems which elements depend on parameters varying within given intervals. 


\section{Mathematica Software, Numerical Examples}

Methods and algorithms discussed in this paper are implemented in the environment of Mathematica [10]. The Mathematica package IntervalComputations 'LinearSystems' contains a collection of functions which compute guaranteed inclusions for the solution set of a square interval linear system [5]. The particular solvers differ upon the type of the linear system to be solved and the implemented solution method. Now, this package is extended by functions that find guaranteed enclosures to the solutions of over- and underdetermined real linear systems which do or do not involve parameter dependencies.

ILinearSolve [A, b] computes guaranteed bounds for the solution sets of a square interval linear system and for the solution sets $(6),(7)$, where all elements vary independently in their intervals. The input elements can be either numerical intervals or elements from the domain Real.

ParametricSSolve [Ap, bp, parLst] computes guaranteed enclosures for the solutions of over- and underdetermined real linear systems whose elements depend on interval parameters. The parameters and their interval values should be specified by a list of rules parLst. Mathematica [10] allows convenient symbolic description of the parametric matrix and the right hand side vector.

All the iterative solvers can take options affecting the computational process and/or the output of the particular function. The three options, associated with everyone of the iterative solvers are InnerEstimation, Refinement, and Statistics. InnerEstimation, when set to True, specifies the computing of component-wise inner approximation of the solution set in addition to the outer enclosure. Inner estimations allow to obtain the very important measure for the degree of sharpness of an outer solution set enclosure [7]. Refinement set to True implies the application of an iterative refinement procedure for the outer solution set approximation. Statistics set to True implies output of the number of iterations and the relative improvement by the refinement procedure. The three options are set to False by default.

A web access to the above solvers for interval linear systems can be found at http://cose.math.bas.bg/webComputing/.

Example 1. Consider the $(6 \times 5)$-matrix $M_{3}$, taken from [11, p.143], with rank=4 and depending on a real parameter $a$. The following matrix $A(a)$ with full rank is obtained by deleting the fourth column in $M_{3}$. The right-hand side vector $b$ is constructed so that for every $a$ the exact solution of $A(a)^{\top} A(a) x=A(a)^{\top} b$ is $e x=(1,-1,1,-1)^{\top}$ (see [12]), thus

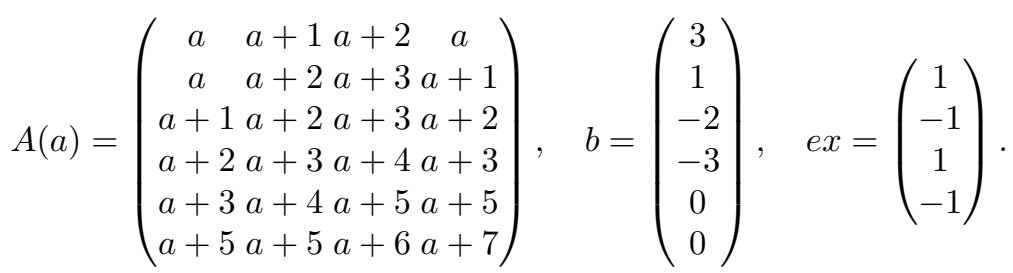

Matrix $A(a)$ is ill-conditioned with $\operatorname{cond}_{\infty}(A) \approx 2602$ for $a=1.0$. We solve the system $[A] x=b$, where $[A]=\diamond(\{A(a) \mid a \in[0.999,1.001]\})$ by the improved 
algorithm and get the following inclusion intervals

$$
\begin{aligned}
& \diamond\left(\Sigma_{m>n}\right) \subseteq \\
& ([0.8629,1.1371],[-1.8273,-0.1727],[0.4924,1.5076],[-1.2742,-0.7257])^{\top} .
\end{aligned}
$$

The linear solver for the corresponding augmented square system which does not accounts for the particular matrix structure gives a solution enclosure overestimating the enclosure (16) by $6-10 \%$.

Example 2. We solve an overdetermined parametric system $A(a) x=b$, where $A(a)$ and $b$ are defined in Example 1, and $a \in[0.99,1.01]$. Our parametric solver found in 1 iteration a sharp enclosure (the numbers have 16-digits mantissa)

$$
\begin{aligned}
& ([0.9 \ldots 97440,1.0 \ldots 02573],[-1.0 \ldots 016600,-0.9 \ldots 983394], \\
& \quad[0.9 \ldots 989643,1.0 \ldots 010354],[-1.0 \ldots 05294,-0.9 \ldots 94715])^{\top}
\end{aligned}
$$

of the exact solution $e x=(1,-1,1,-1)^{\top}$ which actually does not depend on $a$.

The main application of the improved methods and algorithms presented in this paper is for parameter identification in approximation and interpolation when there are errors (uncertainties) in both the output and the variables.

\section{References}

1. Hölbig, C.; Krämer W.: Selfverifying Solvers for Dense Systems of Linear Equations Realized in C-XSC. Universität Wuppertal, Preprint BUGHW-WRSWT 2003/1, 2003. (http://www.math.uni-wuppertal.de/wrswt/literatur.html)

2. Krämer W.; Kulisch, U.; Lohner, R.: Numerical Toolbox for Verified Computing II - Advanced Numerical Problems. Universität Karlsruhe, 1994. (http://www. uni-karlsruhe.de ${ }^{\sim}$ Rudolf.Lohner/papers/tb2.ps.gz)

3. Moore, R.: Methods and Applications of Interval Analysis. SIAM, Philadelphia, 1979.

4. Popova, E.D.; Krämer, W.: Parametric Fixed-Point Iteration Implemented in CXSC, Preprint 2003/03, WRSWT, Universität Wuppertal, 2003.

5. Popova, E.: Parametric Interval Linear Solver. Numerical Algorithms, 37 (2004) 1-4, 345-356.

6. Rump, S.: Solving Algebraic Problems with High Accuracy. In: Kulisch, U. and Miranker, W. (eds.): A New Approach in Scientific Computation. Academic Press (1983) 51-120.

7. Rump, S. M.: Rigorous Sensistivity Analysis for Systems of Linear and Nonlinear Equations, Mathematics of Computation 54, 190, (1990), 721-736.

8. Rump, S. M.: Verification Methods for Dense and Sparse Systems of Equations, in Topics in Validated Computations, J. Herzberger, ed., Elsevier Science B. V. (1994), 63-135.

9. Stewart G.H.: Introduction to Matrix Computations. Academic Press, N.Y., 1973.

10. Wolfram, S.: The Mathematica Book, Wolfram Media/Cambridge U. Press, 1999.

11. Zielke, G.: Report on test matrices for generalized inverses, Computing 36 (1986) 105-162.

12. Zielke, G.; Drygalla, V.: Genaue Lösung linearer Gleichungssyteme, Mitteilungen der GAMM 26 (2003) Heft 1/2, 7-107. 